\title{
Quantum Statistical Corrections to Intrabeam Scattering Rates
}

\author{
Andreas Kabel \\ Stanford Linear Accelerator Center, \\ 2575 Sand Hill Road, Menlo Park, CA 94025 \\ E-Mail: andreas.kabel@slac.stanford.edu
}

\begin{abstract}
Intrabeam scattering (IBS) is a relaxation process which brings a bunch of charged particles in an accelerator closer to its thermal equilibrium distribution[1,2]. This leads to a time variation of collective quantities of the bunch, most notably of the emittance. The typical relaxation times can be calculated using the formalism of Bjorken and Mtingwa[2]. This formalism treats the particles in the bunch as distinguishable classical particles. In this paper, we extend the results of [2] to include effects due to quantum statistics. For fermions, the relaxation times will increase due to restricted accessibility of phasespace, while we find a decrease in relaxation times for bosons. The correction factor, however, will be of the or$\operatorname{der} N_{\text {Particles }} \hbar^{3} / p_{0}^{3} \varepsilon_{x} \varepsilon_{y} \varepsilon_{z}$ and will be negligible for today's machines.
\end{abstract}

\section{INTRODUCTION}

In the usual parameter regime of accelerator physics, it is justified to treat a charged particle bunch as a hot gas of classical particles.

In the context of new cooling mechanisms, such as the one proposed in [3], however, one has to consider systems in which the single-particle action can become close to $h$. If we consider these very cold states, quantum phase space granularity becomes an issue.

The zero-temperature ground state of an charged beam in a strong-focusing lattice can be explicitly constructed[4, 5] as a Fermi condensate in a self-consistent mean-field. For $T=0$, it is clear that no scattering processes take place. For small, but finite temperatures, only the particles close to the Fermi surface can scatter off each other.

From this, it is clear the usual intrabeam scattering calculations cannot apply to the low-temperature regime, as they assume that particles are distinguishable and can share phase space cells, leading to a Maxwell distribution in equilibrium. From the explicit construction, however, we know the equilibrium distribution has to be a Fermi distribution. Thus, the usual kinetic equations for IBS need to be modified for a temperature range between Fermi condensation (which suppresses scattering processes) and very low phasespace densities (in which case the classical approximation of a hot gas is applicable).
In this paper, we will generalize the kinetic equation used by Bjorken and Mtingwa ([2], we will frequently refer to their results and formalism) to the quantum case. Their equation approximates the scattering processes by binary collisions. The quantum equivalent of this is a collision integral in the Born approximation, namely, the UehlingUhlenbeck collision integral ${ }^{1}[6,7]$ :

$$
\begin{aligned}
J\left(\boldsymbol{p}_{1}\right)= & \int \mathrm{d}^{3} p_{2} \mathrm{~d}^{3} p_{1}^{\prime} \mathrm{d}^{3} p_{2}^{\prime} \boldsymbol{\delta}\left(\varepsilon_{1}+\varepsilon_{2}-\varepsilon_{1^{\prime}}-\varepsilon_{2^{\prime}}\right) \\
& \left|V\left(\boldsymbol{p}_{1}, \boldsymbol{p}_{2}, \boldsymbol{p}_{1}^{\prime}, \boldsymbol{p}_{2}^{\prime}\right)\right|^{2} \\
& {\left[\left(1+\xi f\left(\boldsymbol{p}_{1}\right)\right)\left(1+\xi f\left(\boldsymbol{p}_{2}\right)\right) f\left(\boldsymbol{p}_{1}^{\prime}\right) f\left(\boldsymbol{p}_{2}^{\prime}\right)\right.} \\
& \left.-\left(1+\xi f\left(\boldsymbol{p}_{1}^{\prime}\right)\right)\left(1+\xi f\left(\boldsymbol{p}_{2}^{\prime}\right)\right) f\left(\boldsymbol{p}_{1}\right) f\left(\boldsymbol{p}_{2}\right)\right],
\end{aligned}
$$

where $f$ is the phasespace density, $\varepsilon_{p}$ is the kinetic energy, $V$ is the interaction matrix element, $J(p) \propto \frac{\mathrm{d}}{\mathrm{d} t} f(p)$, and $\xi$ sets the statistics: $\xi=0$ for Boltzmann, $\xi \stackrel{\text { a }}{=} 1$ for Bose, and $\xi=-1$ for Fermi statistics. The interaction is assumed to be isotropic and translationally invariant. Furthermore, $V$ is assumed to be properly (anti-)symmetrized for bosons (fermions), i. e., it contains both the direct and the exchange amplitude. We treat everything in the beam's frame of reference.

We are interested in the time development of moments of the outer momentum $\boldsymbol{p}_{1}$, so we calculate the quantity $J^{i k}=\int \mathrm{d}^{3} p_{1} J\left(\boldsymbol{p}_{1}\right) p_{1}^{i} p_{1}^{k}$.

\section{CLASSICAL CASE}

Let us first consider the case $\xi=0$; this is the case Bjorken and Mtingwa treated in [2]. In the quantum case, it is sufficient to consider distributions in the momentum variable $\boldsymbol{p}$ only, which can be thought of as reduced Wigner functions $\int \mathrm{d}^{3} q f_{W}(\boldsymbol{q}, \boldsymbol{p})$. We assume them to be Gaussian with correlation matrix $C$, and normalized such that $\int \mathrm{d}^{3} p f(\boldsymbol{p})=N$.

Note that, in the case $\xi=0$, the equilibrium distribution associated with (1) is the Maxwell-Boltzmann distribution $f_{\mathrm{MB}}(\boldsymbol{p}) \propto \exp (-\beta \varepsilon(\boldsymbol{p}))$, leading to $J\left(\boldsymbol{p}_{1}\right)=0$. Setting $\xi= \pm 1$ will give zero growth for $f_{\mathrm{F}, \mathrm{B}} \propto \frac{1}{\exp (\beta(\varepsilon(\boldsymbol{p})-\mu)) \pm 1}$, i. e., the quantum-statistical Fermi and Bose distributions, resp., as one should expect. For the case of a storage ring

\footnotetext{
${ }^{1}$ Everything is in natural units $\hbar=c=1$;

summation is implied over repeated indices
} 
above transition, such an equilibrium distribution does not exist, however, as the hamiltonian is not bounded from below.

In (1), momentum conservation allows us to integrate out one momentum vector and to introduce $\boldsymbol{q}, \boldsymbol{p}_{ \pm}$, where $\boldsymbol{p}_{1,2}=\boldsymbol{p}_{+} \pm \boldsymbol{p}_{-} \pm \boldsymbol{q} / 2$ and $\boldsymbol{p}_{1,2}^{\prime}=\boldsymbol{p}_{+} \pm \boldsymbol{p}_{-} \mp \boldsymbol{q} / 2$. With that, we have $\delta\left(\varepsilon_{1}+\varepsilon_{2}-\varepsilon_{1^{\prime}}-\varepsilon_{2^{\prime}}\right)=\frac{m}{2} \boldsymbol{\delta}\left(\boldsymbol{p}_{-} \cdot \boldsymbol{q}\right)$ and $\mathrm{d}^{3} p_{1} \mathrm{~d}^{3} p_{2} \mathrm{~d}^{3} p_{1}^{\prime} \mathrm{d}^{3} p_{2}^{\prime} \delta_{\text {Scattering }}^{3}=8 \mathrm{~d}^{3} p_{+} \mathrm{d}^{3} p_{-} \mathrm{d}^{3} q$.

Thus,

$$
\begin{aligned}
& J^{i k}=4 m \int \mathrm{d}^{3} p_{+} \mathrm{d}^{3} p_{-} \mathrm{d}^{3} q \delta\left(\boldsymbol{q} \cdot \boldsymbol{p}_{-}\right)|V(|q|)|^{2} \\
& {\left[f\left(\boldsymbol{p}_{+}+\boldsymbol{p}_{-}+\boldsymbol{q} / 2\right) f\left(\boldsymbol{p}_{+}-\boldsymbol{p}_{-}-\boldsymbol{q} / 2\right)-\right.} \\
&\left.\quad-f\left(\boldsymbol{p}_{+}+\boldsymbol{p}_{-}-\boldsymbol{q} / 2\right) f\left(\boldsymbol{p}_{+}-\boldsymbol{p}_{+}+\boldsymbol{q} / 2\right)\right] \\
& \quad\left(\boldsymbol{p}_{+}+\boldsymbol{p}_{-}+\boldsymbol{q} / 2\right)^{i}\left(\boldsymbol{p}_{+}+\boldsymbol{p}_{-}+\boldsymbol{q} / 2\right)^{k}
\end{aligned}
$$

We replace $\boldsymbol{q} \rightarrow-\boldsymbol{q}$ in the second term and shift $\boldsymbol{p}_{-}$by $\boldsymbol{q} / 2$ in both:

$$
\begin{aligned}
& J^{i k}=4 m \int \mathrm{d}^{3} p_{+} \mathrm{d}^{3} p_{-} \mathrm{d}^{3} q|V(|q|)|^{2} \\
& f\left(\boldsymbol{p}_{+}+\boldsymbol{p}_{-}\right) f\left(\boldsymbol{p}_{+}-\boldsymbol{p}_{-}\right) \delta\left(\boldsymbol{q} \cdot \boldsymbol{p}_{-}-\boldsymbol{q} \cdot \boldsymbol{q} / 2\right) \\
& {\left[\left(\boldsymbol{p}_{+}+\boldsymbol{p}_{-}\right)^{i}\left(\boldsymbol{p}_{+}+\boldsymbol{p}_{-}\right)^{k}\right.} \\
&\left.-\left(\boldsymbol{p}_{+}+\boldsymbol{p}_{-}-\boldsymbol{q}\right)^{i}\left(\boldsymbol{p}_{+}+\boldsymbol{p}_{-}-\boldsymbol{q}\right)^{k}\right] .
\end{aligned}
$$

We now use the fact the distribution $f$ is Gaussian with vanishing centers. We can then rearrange the arguments of the $f$ s, giving

$$
\begin{aligned}
& J^{i k}=4 m \int \mathrm{d}^{3} p_{+} \mathrm{d}^{3} p_{-} f\left(\sqrt{2} \boldsymbol{p}_{+}\right) f\left(\sqrt{2} \boldsymbol{p}_{-}\right) \\
& \int \mathrm{d}^{3} q \delta\left(\boldsymbol{q} \cdot \boldsymbol{p}_{-}-\boldsymbol{q} \cdot \boldsymbol{q} / 2\right) V_{0} \frac{p_{-}^{i} q^{k}+p_{-}^{k} q^{i}-q^{i} q^{k}}{|\boldsymbol{q}|^{4}},
\end{aligned}
$$

where we put in the Coulomb interaction $V_{0}|\boldsymbol{q}|^{-4}$. We disregard exchange terms in the matrix element, as simple power-counting shows they will not contribute logarithmically.

The innermost integral $V^{i k}$ can be done by going to spherical coordinates with $\boldsymbol{p}_{-} \| \mathbf{e}_{z}$. Suppressing constant and keeping only logarithmically divergent terms, we get

$$
V_{i k}=-\pi \frac{\left|\boldsymbol{p}_{-}\right|^{2} \delta^{i k}-3 p_{-}^{i} p_{-}^{k}}{\left|\boldsymbol{p}_{-}\right|^{3}} \log ,
$$

where $\log =\int \mathrm{d}\left(\cos \vartheta_{\boldsymbol{p}_{-}, \boldsymbol{q}}\right) / \cos \vartheta_{\boldsymbol{p}_{-}, \boldsymbol{q}}$ (which has to be interpreted as an appropriately regularized quantity) is the plasma logarithm.

After rescaling $\boldsymbol{p}_{ \pm}$by $\sqrt{2}$, we get the 'diffusion kernel'

$$
J^{i k}=-\frac{m N \pi V_{0}}{\sqrt{2}} \int \mathrm{d}^{3} p f(\boldsymbol{p}) \frac{|\boldsymbol{p}|^{2} \delta^{i k}-3 p^{i} p^{k}}{|\boldsymbol{p}|^{3}} \log
$$

in agreement with the result of Bjorken and Mtingwa [2].

\section{QUANTUM CORRECTIONS}

\subsection{Pauli Blocking}

Now we set $\xi= \pm 1$ in (1). This leads to a correction term

$$
J_{\text {Quantum }}^{i k}=J^{i k}+\xi \Delta J^{i k},
$$

where the $\Delta J^{i k}$ integrals contain all $f^{3}$ terms; the $f^{4}$ terms vanish for all statistics. It is easy to see that the correction is of the order $N J^{i k} / \Gamma$, where $\Gamma$ is the phasespace volume; the correction will lead to an increase (decrease) of the decay times for fermions (bosons).

We use the same variables as above, giving

$$
\begin{aligned}
\Delta J^{i k}= & 8 m \int \mathrm{d}^{3} p_{+} \mathrm{d}^{3} p_{-} \mathrm{d}^{3} q \delta\left(\boldsymbol{q} \cdot \boldsymbol{p}_{-}\right)|V(|q|)|^{2} \\
& {\left[\sum_{\sigma= \pm} f\left(\boldsymbol{p}_{+}+\sigma \boldsymbol{p}_{-}-\sigma \boldsymbol{q} / 2\right)\right] f\left(\boldsymbol{p}_{+}+\boldsymbol{p}_{-}+\boldsymbol{q} / 2\right) } \\
& f\left(\boldsymbol{p}_{+}-\boldsymbol{p}_{-}-\boldsymbol{q} / 2\right)\left(p_{+}^{i}+p_{-}^{i}\right) q^{k} .
\end{aligned}
$$

The difficulty lies in the fact that the quadratic forms in the product distributions do not allow for the elimination of $\boldsymbol{q}$ as in the previous case. Thus, we will keep three Gaussians in the integral. We proceed by shifting variables: $\boldsymbol{p}_{-}:=\boldsymbol{p}_{-}-\boldsymbol{q} / 4$ and $\boldsymbol{p}_{+}:=\boldsymbol{p}_{+}+\sigma \boldsymbol{q} / 4$ :

$$
\begin{aligned}
\Delta J^{i k}= & 8 m \sum_{\sigma= \pm} \int \mathrm{d}^{3} p_{+} \mathrm{d}^{3} p_{-} \\
& f\left(\sqrt{2}\left[\boldsymbol{p}_{+}+\sigma \boldsymbol{p}_{-}\right]\right) f\left(\boldsymbol{p}_{+}-\sigma \boldsymbol{p}_{-}\right) \times \\
& \int \mathrm{d}^{3} q f(\sqrt{2} \boldsymbol{q}) \boldsymbol{\delta}\left(\boldsymbol{q} \cdot \boldsymbol{p}_{-}-\boldsymbol{q} \cdot \boldsymbol{q} / 4\right) \\
& |V(|q|)|^{2}\left(p_{+}^{i}+p_{-}^{i}-(1-\sigma) q^{i} / 4\right) q^{k} .
\end{aligned}
$$

Expanding $f$ in $\boldsymbol{q}$ in the innermost integral, we see that only the zeroth order contributes to leading-log order, so we can replace $f(\sqrt{(} 1 / 2) \boldsymbol{q})$ by $f(0)$, i. e., its normalization constant $\frac{N}{\Gamma}$, where $\Gamma=(2 \pi)^{3} \sqrt{\operatorname{det} C}$ is the phasespace volume of the bunch's six-dimensional one- $\sigma$ ellipsoid.

We now can integrate out $\boldsymbol{p}_{+}$. We need the following integrals:

$$
\begin{aligned}
K_{0} & =\int \mathrm{d}^{3} p_{+} f\left(\sqrt{2}\left[\boldsymbol{p}_{+}+\sigma \boldsymbol{p}_{-}\right]\right) f\left(\boldsymbol{p}_{+}-\sigma \boldsymbol{p}_{-}\right) \\
& =3^{-\frac{3}{2}} N f\left(\sqrt{10 / 3} \boldsymbol{p}_{-}\right)
\end{aligned}
$$

and

$$
\begin{aligned}
K_{1}^{i} & =\int \mathrm{d}^{3} p_{+} f\left(\sqrt{2}\left[\boldsymbol{p}_{+}+\sigma \boldsymbol{p}_{-}\right]\right) f\left(\boldsymbol{p}_{+}-\sigma \boldsymbol{p}_{-}\right) p_{+}^{i} \\
& =-\sigma 3^{-\frac{5}{2}} N f\left(\sqrt{10 / 3} \boldsymbol{p}_{-}\right) p_{-}^{i} .
\end{aligned}
$$

The reduced distribution function is independent of $\sigma$, so all terms $\propto \sigma$ drop out of the sum.

The innermost integral is proportional to $V_{i k}$, so

$$
\frac{\Delta J^{i k}}{J^{i k}}=\frac{N}{\Gamma} \frac{3^{-3 / 2} \int \mathrm{d}^{3} p_{-} f\left(\sqrt{10 / 3} \boldsymbol{p}_{-}\right) V_{i k}}{2^{-3 / 2} \int \mathrm{d}^{3} p_{-} f\left(\sqrt{2} \boldsymbol{p}_{-}\right) V_{i k}}=\frac{2 N}{5 \Gamma} \sqrt{\frac{2}{3}}
$$




\subsection{Exchange Term}

Another term neglected in the classical approximation[2] is the exchange term in the interaction matrix element. As it will not contribute to leading-log order, this is justified. However, if $\frac{\Gamma}{N} \approx \log$, this term becomes important in our consideration. We can proceed as in section 2 up to (2); the exchange term will give a contribution

$$
\begin{array}{r}
V_{\text {Exchange }}^{i k}=\xi \int \mathrm{d}^{3} q \delta\left(\boldsymbol{q} \cdot \boldsymbol{p}_{-}-\boldsymbol{q} \cdot \boldsymbol{q} / 2\right) \\
V_{0} \frac{p_{-}^{i} q^{k}+p_{-}^{k} q^{i}-q^{i} q^{k}}{2|\boldsymbol{q}|^{2}\left|\boldsymbol{p}_{-}-\boldsymbol{q} / 2\right|^{2}}
\end{array}
$$

which stems from the mixed terms in the transition probability

$$
P=\left|\left\langle\boldsymbol{p}_{1}^{\prime}, \boldsymbol{p}_{2}^{\prime}|V| \boldsymbol{p}_{1}, \boldsymbol{p}_{2}\right\rangle+\xi\left\langle\boldsymbol{p}_{1}^{\prime}, \boldsymbol{p}_{2}^{\prime}|V| \boldsymbol{p}_{2}, \boldsymbol{p}_{1}\right\rangle\right|^{2} .
$$

Again, this integral is easily evaluated in spherical coordinates, giving

$$
V_{\text {Exchange }}^{i k}=\xi \frac{\text { const }}{2 \log } V^{i k} .
$$

The non-logarithmic parts of the direct terms (i.e., the terms neglected in (3) are of the same order, they read

$$
V_{\text {Direct, nolog }}^{i k}=-\frac{\text { const }}{\log } V^{i k},
$$

where const $=\int \mathrm{d}\left(\cos \vartheta_{\boldsymbol{p}_{-}, \boldsymbol{q}}\right) \cos \vartheta_{\boldsymbol{p}_{-}, \boldsymbol{q}}$ is a finite integral whose boundaries are subject to the same cutoff as the ones of the log integral.

\subsection{Total Correction}

We have found that all correction terms are proportional to the same tensor. Thus, the formalism for locally evaluating the quantities $J_{i k}$ remains applicable; all integrands in the ring-averaged quantities in [2] merely need to be augmented by a factor of (in usual units)

$$
1+\xi \frac{4 N \hbar^{3}}{5 \sqrt{6 \operatorname{det} C_{\mathrm{Lab}}}}+(\xi-2) \frac{\text { const }}{2 \log },
$$

where $C_{\mathrm{Lab}}$ is the correlation matrix of positions and momenta in the laboratory frame. The second term is temperature-dependent, as one would expect, lowering the scattering rates for fermions as their phasespace volume shrinks.

\section{ACKNOWLEDGMENTS}

I wish to thank M. Venturini for helpful discussions.

\section{REFERENCES}

[1] A. Piwinski. Intrabeam scattering. (talk). 1974. In *Stanford 1974, Proceedings, Ninth International Conference On High Energy Accelerators*, Springfield 1975, 405-409 and Hamburg Desy - INTERNAL REPORT H1-73-3 (73,REC.FEB 74) $17 \mathrm{p}$.

[2] James D. Bjorken and Sekazi K. Mtingwa. Intrabeam scattering. Particle Accelerators, 13:115, 1983.

[3] Zhiron Huang. Radiative Cooling of Relativistic Electron Beams. PhD thesis, Stanford University, 1999.

[4] A. Kabel. Collective quantities of a fermionic particle beam in a circular accelerator near zero temperature. submitted to Phys. Rev. E, 2001.

[5] A. Kabel. Quantum ground state and minimum emittance of fermionic particle beam in a circular accelerator. In Proceedings of the Conference on Quantum Aspects of Beam Physics, Capri, Italy, 15-20 October 2000, 2000. (submitted).

[6] G. Uhlenbeck and E. Uehling. Phys. Rev., 43:552, 1933.

[7] Dmitrii Zubarev, Vladimir Morozov, and Gerd Röpke. Statistical Mechanics of Nonequilibrium Processes, volume 1. Akademie Verlag, 1996. 